\title{
Comprehensive Learning on Characteristics, Applications, Issues and Limitations of Manets
}

\author{
S. John Justin Thangaraj, Rengarajan A, Selvanayaki S
}

\begin{abstract}
This paper is aimed at giving an insight view over the research domains across various levels of Mobile Ad-hoc Networks. A mobile ad-hoc sensor network follows a broader sequence of operational scenarios, unlike typical sensor networks that communicate with the centralized controller. Hence they demand a less complicated setup favorably. Mobile ad-hoc sensors are otherwise known as hybrid ad-hoc networks that consist of some sensors spreads in a geographical area. All individual sensors are capable of mobile communication and have some level of intelligence to process signals and to transmit information. Mobile ad-hoc sensor networks are very constructive in different circumstances. These networks advance the operational efficiency of individual civilian applications. Mobile ad-hoc sensor network becomes highly adaptable so that it can be deployed in almost all environments.The evolution of research in MANET has been started from routing of packets, error free routing, reliable route to stable and secure path of communication in present days. Recent researchers may have much opening in the space of security over the data transmission in the MANET.
\end{abstract}

\section{INTRODUCTION}

The research by Defence Advanced Research Projects Agency (DARPA) on MANETs was released after 1973. The research was first begun to initiate the packet-switched radio communications providing reliable computer communications.

The first generation ad-hoc networks were designed and used for military communication scenarios and were made up of packet radio network components. The design possessed the firmware loaded into the packet radio either locally through the serial interface or from the packet radio network. The firmware in each packet radio gathers information about nodal capacity, and route characteristics and provides this knowledge to debugging and monitoring. The radio frequency technology was used to transmit and receive data according to the physical, data link and network layer of the Open Systems Interconnection (OSI) model.

The second generation of the ad-hoc networks prevailed from the early 1980's to the mid-1990. The purpose of installation and use was similar to that of the first generation ad-hoc networks, and they were used for aiding contest operations. The focus of the second generation ad-hoc networks was only the further advancement of the

Revised Manuscript Received on July 18, 2019.

S. John Justin Thangaraj, Associate Professor, ' Saveetha School of Engineering, Saveetha Institute of Medical and Technical Sciences, Chennai, Tamil Nadu, India.

Rengarajan A, Professor, Vel Tech Multi Tech Dr.Rangarajan Dr.Sakunthala Engineering College, Chennai, Tamil Nadu, India.

Selvanayaki S, Assistant Professor, Vel Tech Multi Tech Dr.Rangarajan Dr.Sakunthala Engineering College, Chennai, Tamil Nadu, India. previously built ad-hoc network structure. Some of the second generation developments include Global Information Systems and Near-Term Digital Radio. The survivable adaptive radio networks program was designed in the years 1989 to 1983.This was also an effort to provide packet radio switched networking to the mobile battlefield elements in infrastructure-less environments.

The third generation MANET is a revived form of second generation ad-hoc networks. With the increase in the number of computers and other communication devices, the ad-hoc technology was used for many commercial reasons. The idea of introducing mobility in the nodes was first proposed in research conferences, following which the MANETs came into existence. The MANET was hence recognized and revived as a potential technology. The commercial applications of the third generation ad-hoc networks included Bluetooth and ad-hoc sensors.

\section{MANET ARCHITECTURE}

MANET has no fixed structure; however, the communication paradigm has followed the OSI network model. The general relationship between nodes is shown in figure 1.1. Each node is capable of acting both as a client and a server and switches operations depending on the current requirement. Also, every node is capable of decision making depending on whichever choice of action can give the best outcome of the communication process.

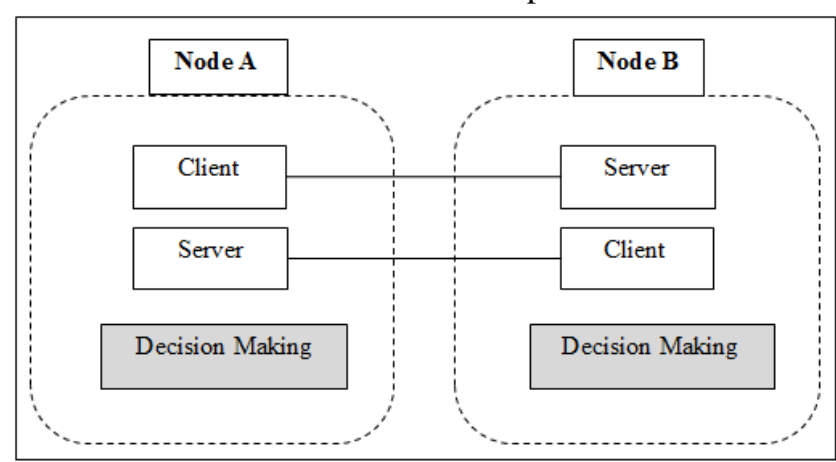

Figure 1.1 Architecture of a MANET

\section{MANET CHARACTERISTICS \& RESULTS}

MANET possesses unique characteristics. Some of the features are explained in the following section.

- Decentralized architecture: All nodes in the network are independent and can configure themselves autonomously into a network. Each node is free to make a 


\section{COMPREHENSIVE LEARNING ON CHARACTERISTICS, APPLICATIONS, ISSUES AND LIMITATIONS OF MANETS}

decision on whether to forward or drop the data packets while routing. In other words, nodes possess independent computational, switching and communication capabilities. nodes distributed in the network area are not governed by a central body.

- Neighbour Discovery: Each node in a MANET is capable of discovering its neighbors by sending wireless signals. The fundamental principle behind the MANET is the discovery of neighbours that are within the direct communication range for a node.

- Heterogeneity: MANET can be formed by some nodes that are heterogeneous in nature. For example, it is possible to create an ad-hoc network between a laptop, palmtop, smart phone, smart sensor and an iPod similar to that of forming the network between a couple of laptops.

- Low powered: All nodes in a MANET are powered by batteries. A similar network is represented by the nodes that possess same battery sizes, whereas heterogeneous MANET consists of nodes with different battery sizes. Efficient utilization of battery power for node operations like transmit, receive and displacement results in the performing network maneuver for longer durations of time.

- Dynamically Changing Topology: Since the they move, their topology is said to by dynamically changing. In spite of the dynamically changing topology, successful data transmission can be observed in the nodes. One of the first dynamical topological changes and adaptability was found in MANETs (Yang et al, 2006).

- $\quad$ Seamless Interaction: One of the most vital needs for the operation of MANETs is the seamless connectivity provided by the nodes once a link is established. It is possible to maintain seamless connectivity between the devices even during node mobility.

- $\quad$ Fluctuating Link Capacity: The capacity of the wireless links is never constant because of the many losses caused by environmental factors and fading. Orthogonal Frequency Division Multiplexing (OFDM) is used in the physical layer of the MANETs.

\section{MANET CHALLENGES}

The following are some of the core routing issues to be considered while positioning MANETs.

- Unpredictability of Environment: The wireless environment is not defined or constrained by any wireless medium. Wireless signals are usually obstructed and have trouble penetrating into solid objects which can be any numbers of things such as hills, buildings, single walls or even people. Including the case for the Line Of Sight (LOS) operation where there is no obstacle in between the end terminals, the communication efficiency is never ideal. The more the obstructions between the transmitter and receiver, the more are the chances for the signal strength to be affected. Although it is not practical to have an LOS operation of terminals in the real world, it is possible to minimize the effect it will have by using specific frequencies available in the spectrum. This architecture is called as decentralized because the nodes arrange and configure themselves simultaneously as

- Unreliability of Wireless Medium: Wireless medium is more prone to errors due to much interference in the common shared medium. The interferences caused by the adjacent frequencies significantly reduce the performance of the wireless networks.

- $\quad$ Resource Constrained Nodes: Wireless Networks allow more than one person to communicate with another network source at any one time. Instantaneously new nodes should be added on to the network, which means the available bandwidth will be utilized by some nodes that will be simultaneously communicating. The resources constrained in the nodes are the battery, bandwidth of communication, processing and computational memory and any other additional storage.

- $\quad$ Node Failures: In case a node fails due to lack of battery power, continuing data transfer becomes an issue. Failure of intermediate nodes during data transmission is one important research issue that is under investigation. Such failures can be avoided by proper energy management and prediction mechanisms, in worst cases, they can be handled using recovery mechanisms.

- $\quad$ Congested Nodes or Links: MANET has a fixed range of bandwidth that has to be utilized by all the operating nodes. Hence the nodes are limited by the bandwidth they use. Wireless nodes need to use the same media for communication, and therefore they naturally contend to use the available links in the bandwidth. This causes the links to be congested when too many nodes in a locality are communicating simultaneously.

- Transmission Errors: Some transmission errors are caused in the wireless medium. If a part of the signal is affected by the environmental factors, then some possible mistakes are found in the signal received at the receiver end. Proper error detection and correction mechanisms are to be incorporated in the first networking layers.

- Dynamic Topology: The ad-hoc nature of the typical wireless networks forms a dynamic topology with multi hop communication, randomly changing topology, and unpredictable bidirectional links leads to great challenges to the researchers of MANET.

\section{MANET MULTI-LAYER ISSUES}

All networks follow the standard OSI model in the design and operation. Ad-hoc networking can be termed as a multilayer problem because of the various challenges faced at every layer as listed:

Layer 1: Physical layer describes how data packet is processed at the networking devices and converted into electrical signals. This layer must acclimate to swift deviations in link features.

Layer 2: Media Access Control (MAC) layer which is the sub-layer of data link layer needs to minimize the occurrences of collisions, allow fair access to the channel and semi-reliably transport data over the shared wireless links even in the existence of rapid changes and hidden or exposed terminal problems.

Layer 3: The network layer needs to determine the path 
towards the destination and distribute the information used to calculate paths in a way that maintains efficiency when links change often, and bandwidth is at a premium quality. It also needs to integrate smoothly with traditional, non ad-hoc aware internetworks and perform self-initiated functions such as auto-configuration in the dynamically changing environment.

Layer 4: The transport layer must have enough capability to handle delay and packet loss statistics that are very much different compared their wired counterparts.

Upper Layers: User Applications need to be designed in such a way to handle frequent disconnection and reconnection with peer applications as well as widely varying delay and packet loss characteristics.

\section{MANET MOBILITY MODELS}

Mobility management patterns for mobile communication systems make use of mobility models for calculating forthcoming user locations. (Sun \& Sauvola, 2002), (Kammann et al, 2003), (Seah et al, 2006) have proposed. Two most widely used mobility models suit the practical applicability of the nodes in a real network. They are random waypoint mobility model and city selection model.

\subsubsection{Random Way Point Mobility Model}

The Random Waypoint Model (Johnson and Maltz, 1998) became the "benchmark" mobility model for evaluating the MANET routing protocols, because of its simplicity and wide availability. This tool is included in the widely used network simulator (Breslau et al, 2000). As an example, the movement trace of a node is shown in figure 1.2.

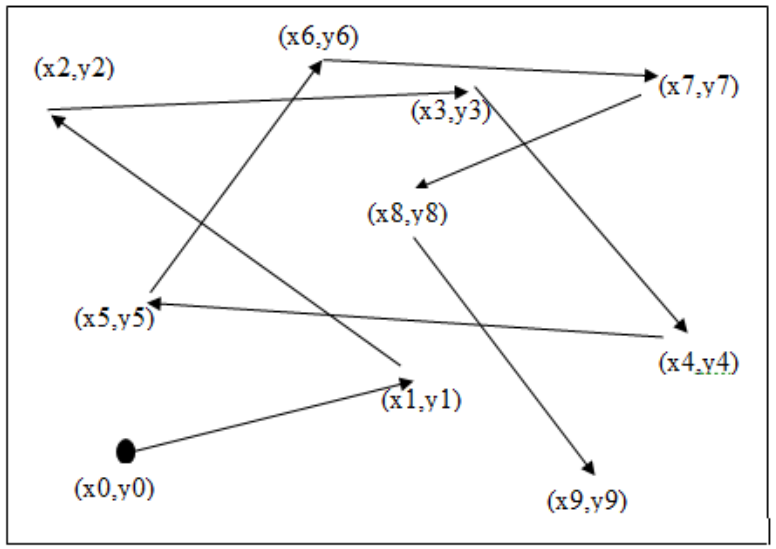

Figure 1.2 Random Way Point Mobility Model

In the NS2 simulation environment, the implementation of this mobility model is created as follows: as the simulation starts, each mobile node randomly selects any one location in the simulation field described as the destination. It then moves towards this destination with persistent velocity chosen uniformly and randomly from $[0$, $\mathrm{V}]$, where the parameter $\mathrm{V}$ denotes the maximum permissible velocity for every mobile node. The velocity and direction of a node are chosen autonomously with each other nodes.

\subsubsection{City Section Model}

The City Section Model (CSM) is another model that represents the node movement in a well-built city. In the CSM, the simulation area is a street network that accounts for a section of a town where the ad hoc network exists. The streets and speed limits on the roads are based on the type of city being simulated.

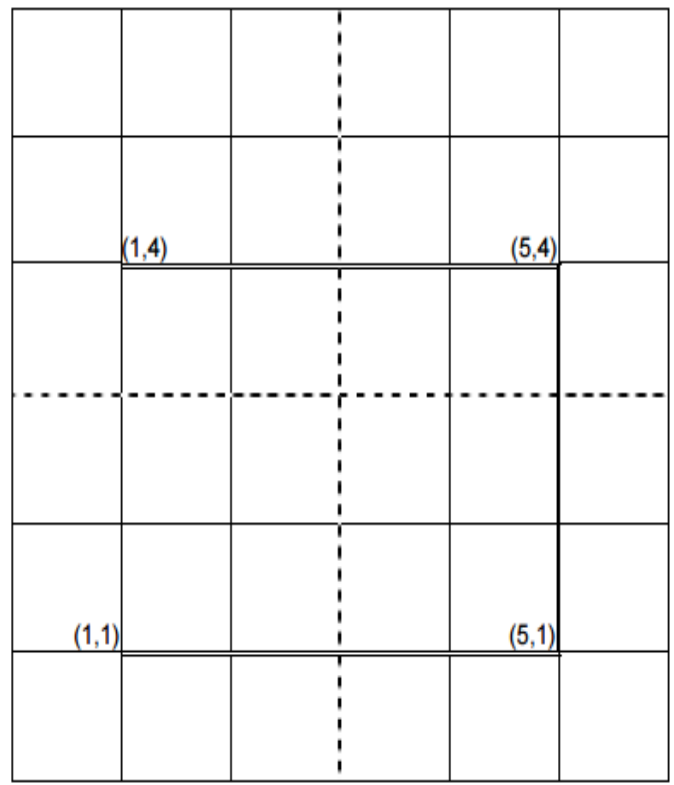

Figure 1.3 City Section Model (Davies, 2000)

Each node begins the simulation at a predefined intersection of two lines making a junction point. A node then randomly chooses a destination, also represented by the crossing points of two streets. Moving to this destination involves one horizontal and one vertical movement. When the destination is reached, the mobile randomly chooses another destination and repeats the same process from the beginning.

Figure 1.3 shows the movements starting at $(1,1)$, moving to $(5,4)$ and then moving to $(1,4)$ that clearly represents node movement in a CSM. Also, people typically tend to travel in similar patterns when driving across town or to walk across campus. Graph-based Mobility Model is similar to City Selection Mobility Model in a way if a city is treated as the graph where all streets are edges of graphs and all possible locations of mobile nodes are vertexes of the graph.

\section{CONCLUSION}

A study over the characteristics and limitations of MANET are done in this paper to understand the various research problems with respect to their operations and the current problem domain. The main problem identified is that there is a need for network reliability in MANET. This work mainly focuses on limitations and security issues for providing reliability in MANET for improved performance. To improve the safe and secure path of the routing system among the nodes, the fairness flow measures have been used for abstraction routing. For the sender and the receiver, this affords a good communication service without the network congestion. But there are large extents for the loss of communication link happens among the intra and inter segment nodes in the network. At last the communication

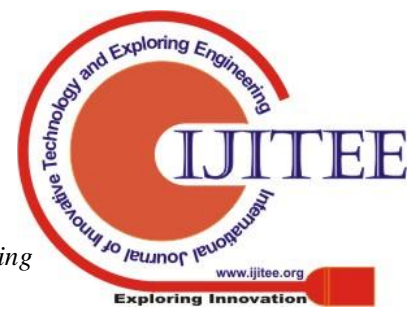


link may prone to failure. To progress the safe communication link between the nodes, better methods can be used in the future to afford an improvement of proficient routing in the ad hoc networks.

\section{REFERENCES}

1. Bamis, A., Boukerche, A., Chatzigiannakis, I. and Nikoletseas, S., "A mobility aware protocol synthesis for efficient routing in ad hoc mobile networks", Computer Networks, Vol. 52, No. 1, pp. 130-154, 2008.

2. Chakeres, I.D. and Perkins, C.E., "Dynamic MANET ondemand routing protocol", IETF Internet Draft, draft-ietfmanet-dymo-12.txt, 2008.

3. Garcia-Luna-Aceves, J.J. and Madruga, E.L., "The core assisted mesh protocol", IEEE Journal on selected Areas in Communications, Vol. 17, No. 8, pp. 1380-1394, 1999.

4. Gerla, M., Chen, L.J., Lee, Y.Z., Zhou, B., Chen, J., Yang, G. and Das, S., "Dealing with node mobility in ad hoc wireless network", Formal Methods for Mobile Computing, Springer Berlin Heidelberg, pp. 69-106, 2005.

5. Hong, X., Xu, K. and Gerla, M., "Scalable routing protocols for mobile ad hoc networks", IEEE network, Vol. 16, No.4, pp. 11-21, 2002.

6. Johnson, D., Hu, Y.C. and Maltz, D., "The dynamic source routing protocol (DSR) for mobile ad hoc networks for IPv4”, No. RFC 4728, 2007.

7. Jubin, J. and Tornow, J.D., "The DARPA packet radio network protocols", Proceedings of the IEEE, Vol. 75, No. 1, pp. 21-32,1987.

8. Kammann, J., Angermann, M. and Lami, B., "A New Mobility Model Based on Maps", Proceedings of the 58th IEEE Semiannual Vehicular Technology Conference, Orlanda, USA, 2003.

9. Lu, J.L. and Valois, F., "On the data dissemination in WSNs", Third IEEE International Conference on Wireless and Mobile Computing, Networking and Communications, WiMOB, pp. 58-58, 2007.

10. Murthy, S. and Garcia-Luna-Aceves, J.J.,“An efficient routing protocol for wireless networks", Mobile Networks and Applications, Vol. 1, No. 2, pp. 183-197, 1996.

11. Perkins, C.E. and Royer, E.M., "Ad-hoc on-demand distance vector routing”, Mobile Computing Systems and Applications, Proceedings, $2^{\text {nd }}$ IEEE Workshop, pp.90100, 1999.

12. Rodoplu, V. and Meng, T.H., "Minimum energy mobile wireless networks", IEEE Journal on selected areas in communications, Vol. 17, No. 8, pp. 1333-1344, 1999.

13. Seah, W.K., Lee, F.W., Mock, K.W. and Kwek, M.Q., "Mobility modelling of rush hour traffic for multi-hop routing in mobile wireless networks", Vehicular Technology Conference, VTC-Fall, IEEE, pp. 1-5, 2006.

14. Sholander, P., Tracey, O. and Paul, C., "Wireless routing protocol for ad-hoc networks", U.S. Patent No. 7,177,295, 2007.

15. Souihli, O., Frikha, M. and Hamouda, M.B., "Loadbalancing in MANET shortest-path routing protocols", Ad Hoc Networks, Vol. 7, No. 2, pp. 431-442, 2009.

16. Sun, J.Z. and Sauvola, J., "Mobility and mobility management: a conceptual framework", Proceeding of 10th IEEE International Conference on Networks, 2002.

17. Thangaraj, S. John Justin and Rengarajan, A. "Unreliable Node Detection by Elliptical Curve Diffie-Hellman Algorithm in MANET", Indian Journal of Science and Technology, Vol. 9, No. 19, pp. 1-6, 2016.

18. Toh, C.K., "Associativity-based routing for ad hoc mobile networks", Wireless personal communications, Vol. 4, No. 2, pp. 103-139, 1997.
19. Xiaochuan, X., Gang, W., Keping, W., Gang, W. and Shilou, J., "Link reliability based hybrid routing for tactical mobile ad hoc network", Journal of Systems Engineering and Electronics, Vol. 19, No. 2, pp. 259267, 2008.

20. Ye, F., Luo, H., Cheng, J., Lu, S. and Zhang, L.,"A twotier data dissemination model for large-scale wireless sensor networks", Proceedings of the 8th Annual International Conference on Mobile Computing and Networking, pp. 148-159,2002. 